\title{
Compromiso del apéndice cecal por linfoma folicular grado 2. Reporte de un caso de presentación clínica atípica
}

\author{
Agustín Virgili, Carlos Ernesto Wendichansky, Sara Gonorazky, Martín Nieva \\ Médicos de planta, Sanatorio El Carmen. Salta, Argentina. \\ Acta Gastroenterol Latinoam 2021;51(1):106-111 \\ Recibido: 31/07/2020 / Aceptado: 06/02/2021 / Publicado online: 22/03/2021 / https://doi.org/10.52787/efyd8813
}

\section{Resumen}

El compromiso del apéndice cecal por linfoma en forma primaria o secundaria es extremadamente infrecuente; los linfomas primarios representan menos del 0,02\% de las neoplasias del tubo digestivo. Presentamos el raro caso de un paciente que debuta con hemorragia digestiva baja como sintoma principal. En la colonoscopia se encuentra una tumoración que impronta sobre la cara posterior del ciego y sangrado por el orificio apendicular. Se confirma tumor apendicular en la tomografia computada multicorte y se realiza hemicolectomia derecha laparoscópica. El paciente evoluciona favorablemente y es dado de alta al $\sigma^{\text {to }}$ día postoperatorio. El informe de anatomía patológica informa infiltración linfoma del apéndice cecal por linfoma folicular grado 2.

Palabras claves. Apéndice cecal, linfoma, hemorragia digestiva baja, laparoscopía.
Correspondencia: Agustín Virgili

Correo electrónico: agustinv9@hotmail.com

\section{Involvement of the Appendix by Grade 2 Follicular Lymphoma. A Case Report of Atypical Clinical Presentation}

\section{Summary}

The involvement of the appendix by lymphomas, both primarily or secondarily, is extremely rare; primary lymphomas account for less than $0.02 \%$ of gastrointestinal neoplasm. We present the rare case of a patient who debuts with low gastrointestinal bleeding as the main symptom. Colonoscopy found an extra mucosal tumour on the posterior aspect of the ceccum and bleeding from the appendicular orifice. Primary appendicular tumour is confirmed on multislice computed tomography and laparoscopic right hemicolectomy is performed. The patient had an uneventful postoperative course and was discharged on the $\sigma^{\text {th }}$ postoperative day. The pathology report shows a infiltration of the cecal appendix by grade 2 follicular lymphoma.

Keywords. Appendix, lymphoma, low gastrointestinal bleeding, laparoscopy.

\section{Introducción}

El compromiso del apéndice cecal por linfomas es una entidad extremadamente rara, tanto que los linfomas primarios representan solo el $1,7 \%$ de todos los tumores primarios del apéndice y solo el 0,015\% de las neoplasias del tubo digestivo. ${ }^{1,2}$ Las neoplasias apendiculares secundarias son también muy infrecuentes, y la vía de infiltración predominante es por invasión desde la serosa peritoneal. ${ }^{3}$ La presentación clínica más frecuente incluye síntomas inespecíficos, como astenia, pérdida de peso y 
dolor abdominal crónico. ${ }^{4}$ Otra forma clínica reportada con cierta frecuencia es la apendicitis aguda, en cuyo caso el diagnóstico del tumor apendicular se realiza durante el acto quirúrgico. ${ }^{56}$ La hemorragia digestiva como forma de presentación solo ha sido reportada una vez en la literatura. ${ }^{7}$ Presentamos el raro caso de un paciente operado por un tumor apendicular, que debutó con hemorragia digestiva baja anemizante, diagnosticado preoperatoriamente por videocolonoscopía y tomografía computada multicorte. El diagnóstico histopatológico final fue infiltración por linfoma folicular grado 2 .

\section{Caso}

Un paciente de sexo masculino de 64 años, sin antecedentes de relevancia, consulta en la central de emergencias por cuadro de hematoquecia de una semana de evolución, asociado a astenia y pérdida de peso. Al examen físico, el paciente no presentaba dolor ni masa palpable abdominal. Al tacto rectal se constata presencia de restos de sangre fresca. El resto del examen físico fue normal.

En el análisis de laboratorio al ingreso se constata anemia, con hemoglobina de $8 \mathrm{~g} / \mathrm{dL}$ y hematocrito de $26 \%$, caracterizada como ferropénica. El resto del laboratorio, normal. Se programa videocolonoscopía, que constata una tumoración tubular que empuja desde la submucosa hacia la luz del ciego en su cara posterior, asociada a sangrado a través del orificio del apéndice cecal (Figura 1). El resto del examen videoendoscópico fue normal.

Figura 1. Videoendoscopia digestiva baja. Se evidencia sangrado hacia la luz del ciego por orificio apendicular.

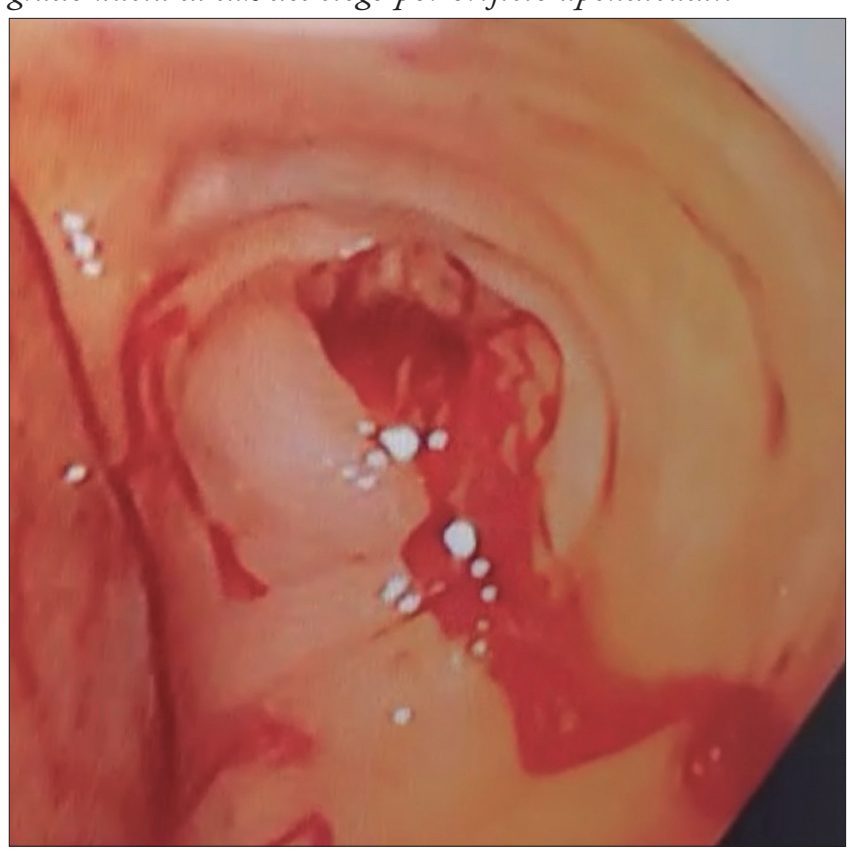

Con sospecha de tumor apendicular, se realiza tomografía computada multicorte de tórax y abdomen. Esta muestra una lesión tumoral bien definida en la fosa ilíaca derecha, en íntimo contacto con la cara posterior del ciego, que se origina en la base del apéndice cecal, con una densidad baja en el centro de esta, lo que sugiere la presencia de un tumor o mucocele apendicular (Figura 2). No se observa neumoperitoneo ni líquido libre en la cavidad abdominal. Se observan adenomegalias en el mesocolon derecho. No hay signos de lesiones secundarias hepáticas o pulmonares.

Se decide conducta quirúrgica. Se realiza laparoscopía exploradora que confirma la sospecha de tumor apendicular localizado, sin ascitis ni implantes en peritoneo (Figura 3). Se realiza lavado y aspiración de la cavidad para citología oncológica. El tumor se encuentra en situación retro-cecal ascendente. Se realiza hemicolectomía derecha laparoscópica, con linfadenectomía del mesocolon derecho hasta el origen de la arteria cólica derecha. En el espesor del mesocolon derecho se objetivan adenomegalias (Figura 4). Se exterioriza la pieza por una incisión mediana de $8 \mathrm{~cm}$ protegida con dispositivo Alexis ${ }^{\circledR}$ (Aplied Medical-EE. UU.). Se confecciona anastomosis latero-lateral con sutura manual.

El paciente tiene una buena evolución postoperatoria, sin complicaciones. Es dado de alta al $6^{\text {to }}$ día postoperatorio, con controles postoperatorios sin eventos adversos.

El informe de anatomía patológica muestra en la macroscopía un tumor sólido que ocupa completamente la luz apendicular y conserva el contorno vermiforme del órgano (Figura 5), de $9 \mathrm{~cm}$ de largo por $3 \mathrm{~cm}$ de diámetro mayor. Se disecaron adenomegalias en el meso del colon, la mayor de $3 \mathrm{~cm}$ de diámetro, contándose dieciséis ganglios en total. Los cortes histológicos del apéndice cecal muestran mucosa conservada, y subyacente a esta se observa una proliferación linfoide de elementos pequeños y medianos clivados (centrocitos) con escasas células grandes (centroblastos), con tendencia a delimitar folículo. En nueve de los dieciséis ganglios disecados se encuentran cambios histológicos de similares características. Las técnicas de inmunohistoquímica muestran positividad de marcadores CD20, PAX5, BCL2. Ki67 de 7\% con distribución dispersa. Hallazgos compatibles con linfoma no Hodgkin tipo B, sugestivo de linfoma folicular grado 2 (Figura 6). Como parte de la estadificación definitiva del linfoma se realizó una nueva tomografía computada multicorte corporal total que descartó otras posibles localizaciones del linfoma. Se realizó además una biopsia de medula ósea por punción aspiración, que informó ausencia de infiltración medular por linfoma. 
Figura 2. Tomografía computada multicorte. En $A, B, C y D$ se evidencia tumor sólido en topografía del apéndice cecal, de $2 \mathrm{~cm}$ de diámetro transverso y $10 \mathrm{~cm}$ de longitud.
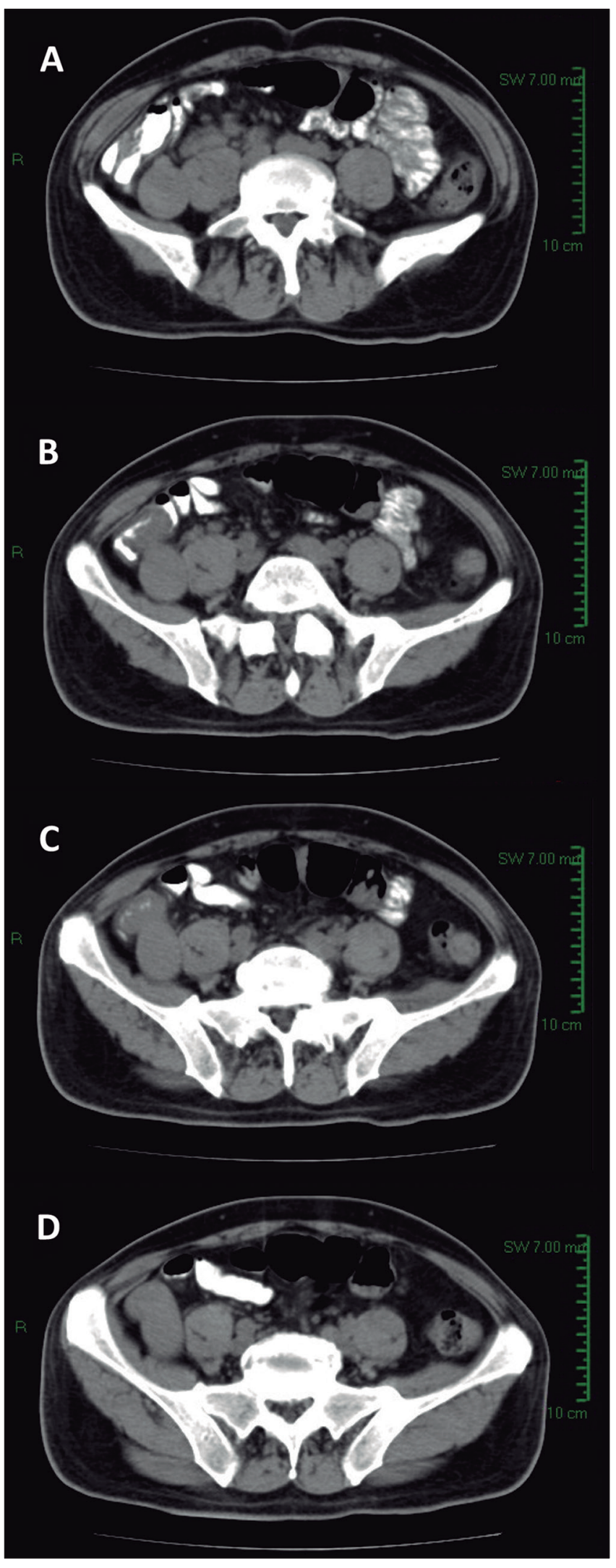

Figura 3. Laparoscopía que muestra tumor apendicular. Se evidencia engrosamiento desde su base en el ciego.

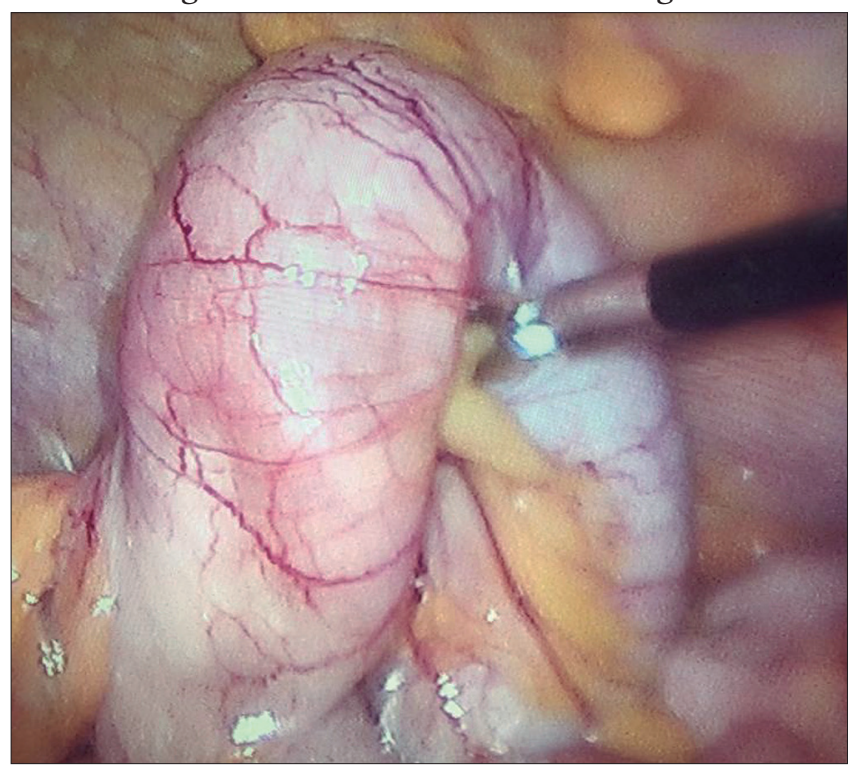

Figura 4. Adenopatías voluminosas en el espesor del mesocolon derecho. Estas se objetivan en la laparoscopia (A) y en la pieza operatoria (B).

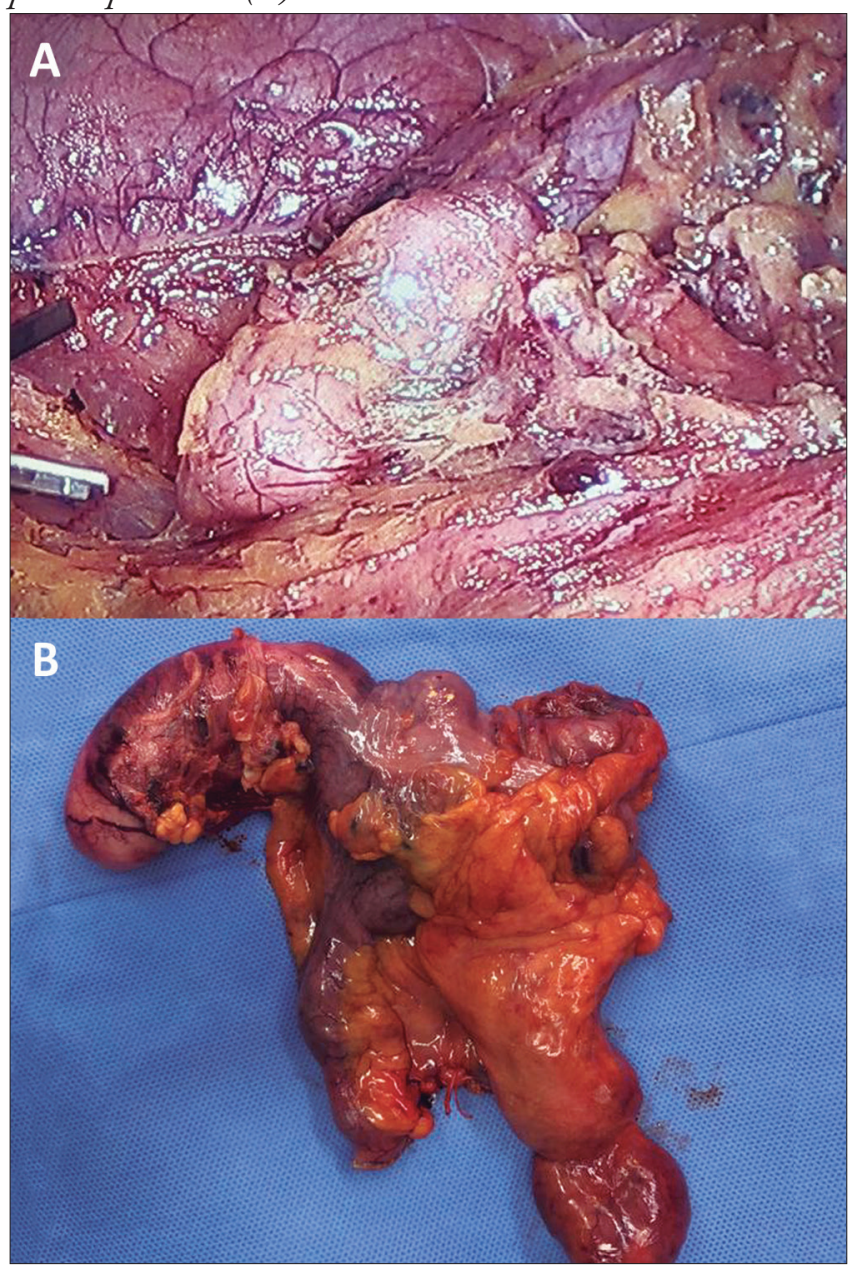


Figura 5. Examen macroscópico de anatomía patológica. Se observa tumor apendicular (A) que ocupa completamente la luz del órgano (B).
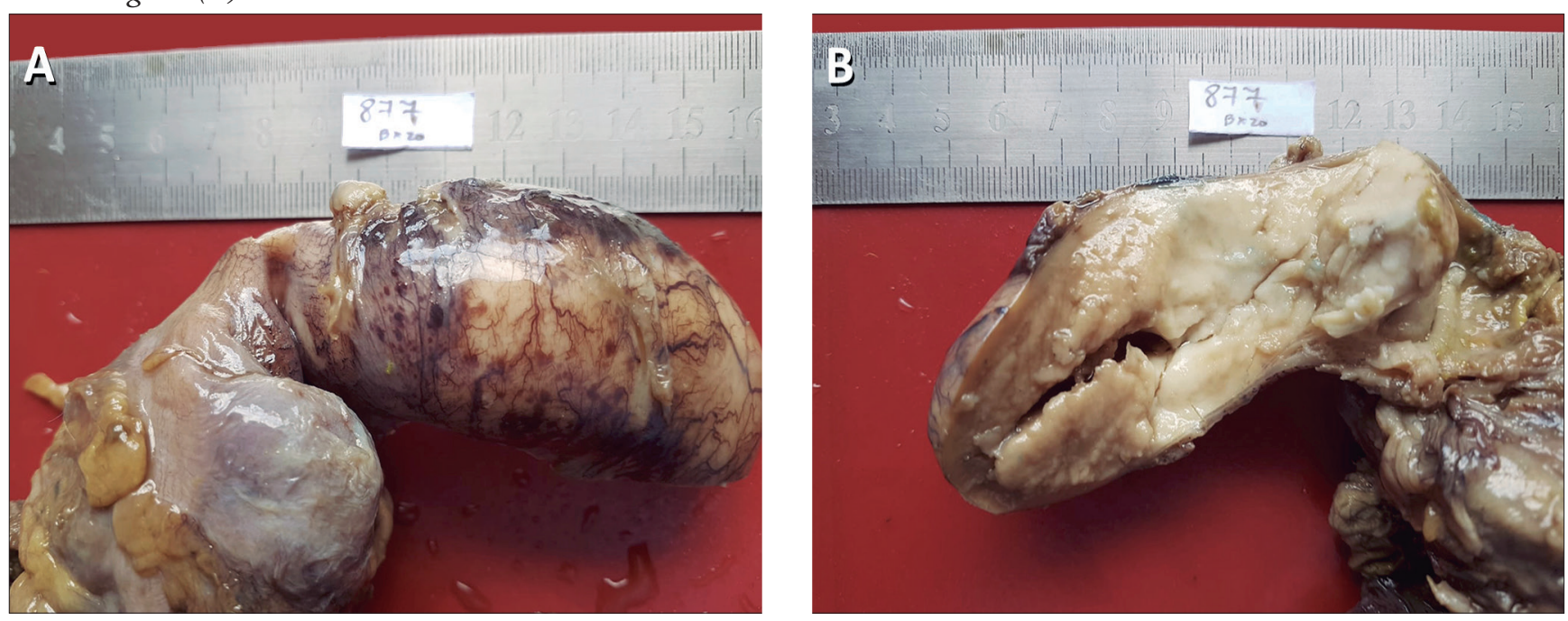

Figura 6. Examen microscópico. La tinción con hematoxilina y eosina muestra una proliferación linfoide de elementos pequeños y medianos clivados (centrocitos) y escasas células grandes (centroblastos) que delimitan folículos (A). Positividad para marcadores inmuno-histoquimicos CD20 (B), PAX5 (C) y BCL2 (D).
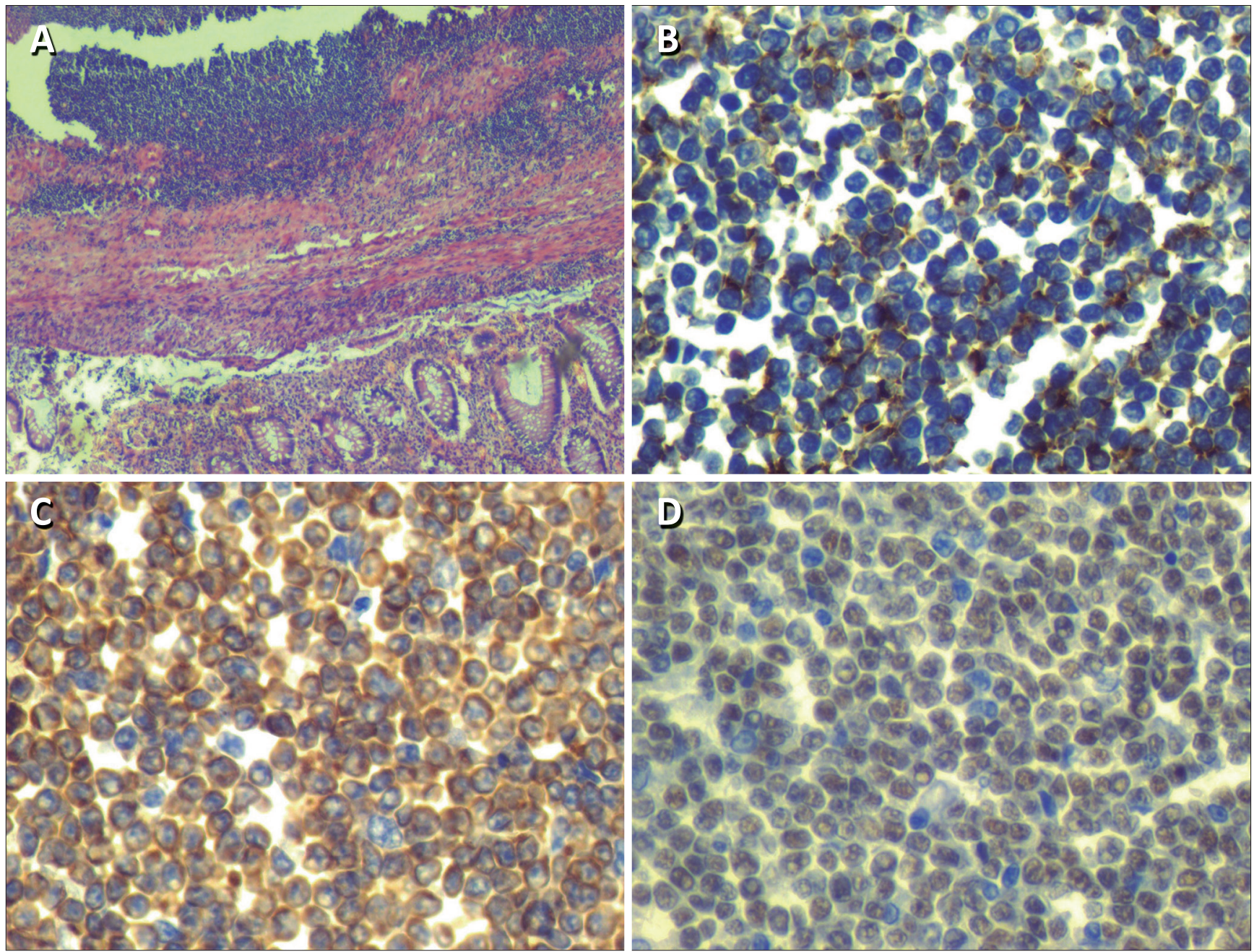


\section{Discusión}

Los tumores del apéndice cecal son una entidad de muy baja incidencia, encontrándose en el 0,9-1,4\% de los especímenes de apendicectomías. ${ }^{8}$ Las neoplasias malignas del tubo digestivo están representadas en su gran mayoría por adenocarcinomas, mientras que los linfomas no superan el $4 \%$ de todas las neoplasias primarias digestivas. ${ }^{9}$ Aun así, el tubo digestivo es el sitio más frecuente de asiento de los linfomas extranodales, y de estos, el linfoma de apéndice solo llega a una incidencia del 2\%. ${ }^{1,2}$

La presentación clínica de los tumores apendiculares es variable. Una forma frecuente de presentación es la apendicitis aguda, en cuyo caso el diagnóstico de tumor apendicular es intraoperatorio o postoperatorio con el reporte de anatomía patológica.5, 6 También puede presentarse como un cuadro de lenta evolución con astenia, pérdida de peso y dolor crónico en fosa ilíaca derecha. En estos casos, el diagnóstico se hace con estudios de imágenes, ya sea ultrasonido o tomografía computada. La hemorragia digestiva como forma de debut es realmente infrecuente, y su diagnóstico endoscópico casi no tiene precedentes en la literatura. En nuestro caso, la videocolonoscopía mostró una masa extramucosa que generaba una impronta tubular en la cara posterior del ciego, con sangrado activo por el orificio apendicular. No hemos registrado publicaciones en la literatura con tal hallazgo.

Desde la introducción y el uso masivo de los métodos de diagnóstico por imágenes como la ecografía y tomografía, el hallazgo de tumores apendiculares ha tenido un ligero ascenso. ${ }^{10}$ No existen patrones de imágenes patognomónicos que permitan diferenciar con certeza la estirpe de una masa apendicular. Si bien una masa apendicular de contenido líquido, entidad conocida como mucocele apendicular, es característica de las neoplasias epiteliales, se han reportado casos de linfomas con características imagenológicas de un mucocele. ${ }^{11}$ En nuestro caso, si bien la masa tumoral era sólida, su baja densidad central en la tomografía computada multicorte obligaba a plantear la posibilidad diagnóstica de un mucocele apendicular.

La conducta quirúrgica y la técnica más adecuada ante el hallazgo de un tumor apendicular sigue siendo motivo de controversia. Sin duda, un aspecto técnico de vital importancia es la adecuada manipulación del tumor, evitando su ruptura, y la protección de la pared abdominal para evitar siembra. El abordaje laparoscópico está validado y es tan seguro como una cirugía abierta. ${ }^{12}$

Se acepta que la apendicectomía es suficiente para lesiones premalignas, carcinoma in situ o tumores neuroendocrinos menores a $1 \mathrm{~cm}$. Para adenocarcinomas y tumores neuroendocrinos mayores a $2 \mathrm{~cm}$ el tratamiento más aceptado es la hemicolectomía derecha con resección del mesocolon correspondiente. Los tumores neuroendocrinos de 1-2 cm se encuentran en una zona gris, en la que entra en juego la presencia o no de adenomegalias y la distancia del tumor a la base apendicular. ${ }^{8}$ Dado que el diagnóstico histológico preoperatorio es excepcional, la mayoría de los linfomas apendiculares son tratados con criterios de neoplasias epiteliales, lo que deriva en cirugías más radicales de lo necesario. En nuestro caso, la base del apéndice cecal se encontraba engrosada, claramente involucrada por tumor; además, las adenomegalias en el mesocolon derecho obligaban a realizar una hemicolectomía derecha con vaciamiento amplio del meso.

Cuando se diagnostica un linfoma apendicular es muy importante la búsqueda exhaustiva de enfermedad en otras localizaciones. Los linfomas de localización atípica son más frecuentes en pacientes inmunocomprometidos, por ejemplo en pacientes VIH positivos. ${ }^{13}$ Nuestro paciente no presentaba inmunodeficiencias y el examen de ELISA para VIH fue negativo. La tomografía computada multicorte corporal total posterior a la cirugía y la punción biopsia de médula ósea no objetivaron cambios compatibles con localizaciones fuera del apéndice y el mesocolon derecho. Por tal motivo, podemos pensar que en este caso se trató de un linfoma primario de apéndice cecal.

\section{Conclusiones}

El compromiso apendicular por linfoma y el linfoma primario de apéndice cecal son entidades patológicas extremadamente raras. Sus formas de presentación clínica más comunes son la de un hallazgo en una apendicitis aguda, o como una masa de crecimiento lento en fosa ilíaca derecha con dolor, astenia y pérdida de peso. La hemorragia digestiva baja como forma de presentación inicial solo tiene escasos reportes y su diagnóstico endoscópico no tiene precedentes en la literatura. La resección por vía laparoscópica de este tipo de tumores es factible y segura. El diagnóstico de linfoma en una pieza de apendicetomía obliga a buscar exhaustivamente la presencia de enfermedad en otras localizaciones y solo en su ausencia podemos aseverar que se trata de un linfoma primario de dicho órgano.

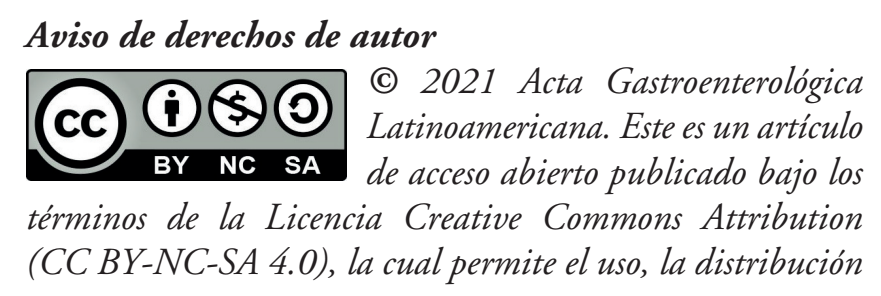


y la reproducción de forma no comercial, siempre que se cite al autor y la fuente original.

Cite este artículo como: Virgili $A$, Wendichansky CE, Gonorazky $S$ y col. Compromiso del apéndice cecal por linfoma folicular grado 2. Reporte de un caso de presentación clinica atipica. Acta Gastroenterol Latinoam. 2021;51(1):106-11. https://doi.org/10.52787/efyd8813

\section{Referencias}

1. Collins DC. 71.000 human appendix specimens. A final report summarizing forty years' study. Am J Proctol. 1963;14:265-81.

2. Gustaffson BI, Siddique L, Chan A, Dong M, Drozdoz I, Kidd $\mathrm{M}$, et al. Uncommon cancers of the small intestine, appendix and colon: an analysis of SEER 1973-2004, and current diagnosis and therapy. Int J Oncol. 2008;33(6):1121-31.

3. Yoon WJ, Yoon YB, Kim YJ, Ryu JK, Kim YT. Secondary Appendiceal Tumors: A Review of 139 cases. Gut and Liver. 2010;4(3):351-56.

4. Ruoff C, Hanna L, Zhi W, Shahzad G, Gotlieb V, Saif MW. Cancers of the appendix: review of the literatures. ISRN Oncol. 2011;2011:728579.
5. Ayub A, Santana-Rodríguez N, Raad W, Bhora FY. Primary appendiceal lymphoma: clinical characteristics and outcomes of 116 patients. J Surg Res. 2017;207:174-80.

6. Guo J, Wu G, Chen X, Li X. Primary appendiceal lymphoma presenting as suspected perforated acute appendicitis: clinical, sonography and CT findings with pathologic correlation. Int J Clin Exp Pathol. 2014;7(10):7068-71.

7. Carpenter BW. Lymphoma of the appendix. Gastrointest Radiol. 1991;16:256-8.

8. Hatch QM, Guilbert EW. Appendiceal Neoplasms. Clin Colon Rectal Surg. 2018;31:278-87.

9. Shiwani MH. Primary malignant lymphoma of the appendix associated with acute appendicitis. J Coll Physicians Surg Pak. 2006;16(1):79-80.

10. Balthazar EJ, Megibow AJ, Gordon RB, Whelan CA, Hulnick D. Computed tomography of the abnormal appendix. Journal of Computer Assisted Tomography. 1988;12(4):595-601.

11. Mori M, Kusunoki T, Kikuchi M, Motoori T, Sugimachi K. Primary malignant lymphoma of the appendix. The Japanese Journal of Surgery. 1985;15:230-3.

12. Bucher P, Mathe Z, Demirag A, Morel Ph. Appendix tumors in the era of laparoscopic appendectomy. Surg Endosc. 2004;18:1063-6.

13. Vannata B, Zucca E. Primary extranodal B-cell lymphoma: current concepts and treatment stategies. Chin Clin Oncol. 2015; $4(1): 10$ 